\title{
Ridge Distance Estimation in Fingerprint Images: Algorithm and Performance Evaluation
}

\author{
Yilong Yin \\ College of Computer Science \& Technology, Shandong University, Shanda South Road 27, Jinan 250100, China
}

Email:ylyin@sdu.edu.cn

Jie Tian

Intelligent Bioinformatics Systems Division, Institute of Automation, The Chinese Academy of Sciences, Beijing 100080, China Email:tian@doctor.com

\author{
Xiukun Yang \\ Identix Inc, One Exchange Place Suite 800, Jersey City, NJ 07302, USA \\ Email:susan.yang@identix.com
}

Received 17 October 2002; Revised 27 September 2003

\begin{abstract}
It is important to estimate the ridge distance accurately, an intrinsic texture property of a fingerprint image. Up to now, only several articles have touched directly upon ridge distance estimation. Little has been published providing detailed evaluation of methods for ridge distance estimation, in particular, the traditional spectral analysis method applied in the frequency field. In this paper, a novel method on nonoverlap blocks, called the statistical method, is presented to estimate the ridge distance. Direct estimation ratio (DER) and estimation accuracy (EA) are defined and used as parameters along with time consumption (TC) to evaluate performance of these two methods for ridge distance estimation. Based on comparison of performances of these two methods, a third hybrid method is developed to combine the merits of both methods. Experimental results indicate that DER is $44.7 \%, 63.8 \%$, and $80.6 \%$; EA is $84 \%, 93 \%$, and $91 \%$; and TC is $0.42,0.31$, and 0.34 seconds, with the spectral analysis method, statistical method, and hybrid method, respectively.
\end{abstract}

Keywords and phrases: fingerprint, ridge distance, spectral analysis, statistical window, hybrid method.

\section{INTRODUCTION}

Fingerprint identification is the most popular biometric technology and has drawn a substantial attention recently [1]. An automated fingerprint identification system (AFIS) includes fingerprint acquisition, feature extraction, fingerprint matching, and/or fingerprint classification. Most AFISs are based on comparison of minutiae, the most prominent being ridge endings and ridge bifurcations [2].

A critical step in automatic fingerprint matching is to extract minutiae automatically and reliably from fingerprint images. Performance of a minutiae extraction algorithm relies heavily, however, on the quality of fingerprint images. In order to ensure a robust performance of an AFIS with respect to quality of fingerprint images, it is essential to incorporate an enhancement algorithm in the minutiae extraction module. Ridge distance is an intrinsic property of fingerprint images and it is used as a basic parameter in fingerprint enhancement in some enhancement methods, during which ridge distance is used to determine the period of enhance- ment mask. It is thus important to be able to estimate ridge distance in fingerprint images reliably in an AFIS.

Fingerprint ridge distance is defined as the distance from a given ridge to adjacent ridges. It can be measured as the distance from the center of one ridge to the center of another, as illustrated in Figure 1. Another notion related to ridge distance is ridge frequency. Ridge frequency is the reciprocal of ridge distance and indicates the number of ridges within a unit length.

Although fingerprint ridge distance is very important in AFIS, it is difficult to estimate due to the following factors:

(1) for the same finger, fingerprint images acquired with different image resolutions may have different ridge distance (s);

(2) even with the same image resolution, noises, such as low contrast, ridge breaks, ridge conglutination, and so forth, may distort estimations;

(3) occurrence of minutiae may disturb the estimation of the ridge distance; 


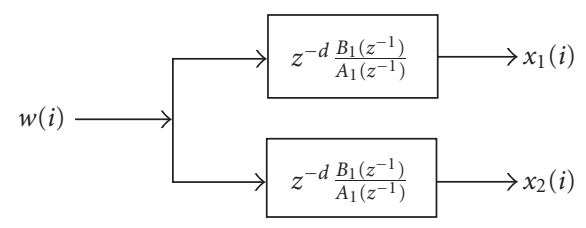

Figure 1: Definition of fingerprint ridge distance.

(4) the existence of high curvature, such as regions containing singularities, makes it difficult to estimate ridge distance in these regions with common methods;

(5) different fingers may have different ridge distances;

(6) within the same fingerprint image, different regions may have different ridge distances.

While problems (3), (4), and (5) are intrinsic properties of fingerprints, problems (1), (2), and (6) may be controlled or improved.

It has been noted above that the ridge distance is important for fingerprint identification. To the best of the authors' knowledge, however, the estimation of ridge distance was only addressed directly in several articles.

O'Gorman and Nickerson [3] used average ridge distance as key parameter in the design of filters. Lin and Dubes [4] attempted to count ridge number automatically. Douglas Hung [5] estimated ridge period over the whole image. Hong et al. [6] presented an oriented window method for estimating fingerprint ridge frequency. This method performs well when ridges in the oriented window have distinct contrast and consistent ridge directions. Kovacs-Vajna et al. [7] brought out geometric and spectral methods to estimate fingerprint ridge distance. One of the merits of the geometric method is that it calculates ridge direction directly, which means it does not depend on the result of ridge direction estimation as the prior procedure. Spectral method divides a fingerprint image into blocks, converts each block of fingerprint image from spatial field to frequency field using discrete Fourier transform (DFT), and estimates ridge distance of a block image according to the distribution of harmonic coefficients. Only less than half of the ridge distances of block images can be estimated directly according to their experimental results, however. Maio and Maltoni [8] did mathematical characterization of the local frequency of sinusoidal signals and developed a two-dimensional model in order to approximate the ridge-line patterns in his method for ridgeline density estimation in digital images.

This paper focuses on ridge distance estimation of fingerprint images. Traditional spectral analysis method is realized and a novel statistical method is presented. Also a hybrid method is brought out and performance evaluation of methods for ridge distance estimation is discussed.

This paper is organized as follows. Traditional spectral analysis method is introduced and applied in Section 2. The statistical method is described in detail in Section 3. Comparison between the spectral analysis method, the statistical method, and a hybrid method is described in Section 4. Performance evaluation and experimental results are shown in Section 5. Section 6 contains the conclusion and discussions.

\section{SPECTRAL ANALYSIS METHOD}

Spectral analysis method is a typical method of signal processing in the frequency field. It transforms the representation of fingerprint images from the spatial field to the frequency field and completes the ridge distance estimation in the frequency field. It is a traditional method for ridge distance estimation in fingerprint images.

If $g_{(x, y)}$ is the gray-scale value of the pixels with coordinates $x, y \in\{0, K, N-1\}$ in an $N \times N$ image, the DFT of $g_{(x, y)}$ is defined as follows:

$$
\begin{aligned}
G_{(u, v)}=\frac{1}{N} \sum_{x=0}^{N-1} \sum_{y=0}^{N-1} g_{(x, y)} e^{-2 \pi j / N\langle(x, y)(u, v)\rangle} & \\
=\frac{1}{N} \sum_{x=0}^{N-1} \sum_{y=0}^{N-1} g_{(x, y)}( & \cos \left(-\frac{2 \pi}{N}\langle(x, y)(u, v)\rangle\right) \\
& \left.\quad+j \sin \left(-\frac{2 \pi}{N}\langle(x, y)(u, v)\rangle\right)\right),
\end{aligned}
$$

where $j$ is an imaginary unit, $u, v \in\{0, \Lambda, N-1\}$, and $\langle(x, y)(u, v)\rangle=x u+y v$ is the vector dot product; $G_{(u, v)}$ is obviously complex. Let $\left|G_{(u, v)}\right|$ denote the magnitude of $G_{(u, v)}$, theoretically speaking,

$$
\begin{aligned}
& \quad\left|G_{(u, v)}\right| \\
& =\frac{1}{N} \sum_{x=0}^{N-1} \sum_{y=0}^{N-1}\left(\left(g_{(x, y)} \cos \left(-\frac{2 \pi}{N}\langle(x, y)(u, v)\rangle\right)\right)^{2}\right. \\
& \left.\quad+\left(g_{(x, y)} \sin \left(-\frac{2 \pi}{N}\langle(x, y)(u, v)\rangle\right)\right)^{2}\right)^{1 / 2},
\end{aligned}
$$

$\left|G_{(u, v)}\right|$ is also called the coefficient. It represents the periodic characteristics of point $u, v$. The dominant period of signals in an area can be determined by analyzing the distribution of values of $\left|G_{(u, v)}\right|$.

A fingerprint image and its DFT at block level with window size of $32 \times 32$ are shown in Figure 2 .

The whole procedure of ridge distance estimation with the spectral analysis method relies on a radial distribution function $Q(r)$ [7] defined as follows:

$$
Q(r)=\frac{1}{\# C_{r}} \sum_{(u, v) \in C_{r}}\left|G_{(u, v)}\right|,
$$

where $0 \leq r \leq \sqrt{2}(N-1), C_{r}$ represents the set of coordinates $u, v$ that satisfy $\sqrt{u^{2}+v^{2}} \approx r$ (approximate equality $\approx$ is resolved by rounding to the nearest integer), and $\# C_{r}$ is the number of elements of $C_{r}$. Based on (3), $Q(r)$ denotes distribution intensity of the signal whose period is $N / r$ in an $N \times N$ image, and the value of $r$ corresponding to the maximum of $Q(r)$ is the incident times of dominant signal in this area. 


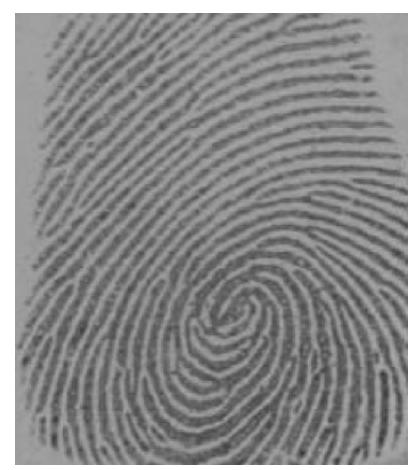

(a)

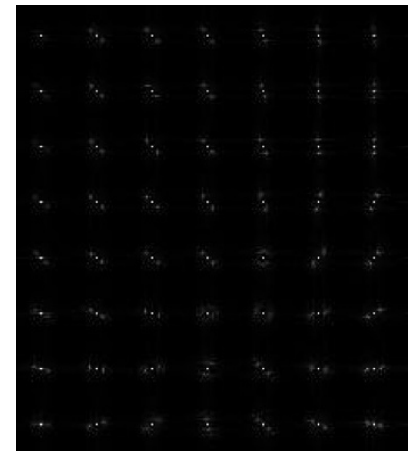

(b)
FIGURE 2: (a) A fingerprint image and (b) corresponding transform result at block level.

The following steps are taken for ridge distance estimation by the spectral analysis method in fingerprint images.

Step 1. Divide a fingerprint into nonoverlap block images of size $N \times N(N=32)$. Each block image is considered as a unit in subsequent procedures.

Step 2. Calculate $\left|G_{(u, v)}\right|$ corresponding to all $g_{(x, y)}, x, y \in$ $\{0, K, N-1\}$, in a block image using DFT.

A two-dimensional fast Fourier transform (FFT) algorithm is applied in this paper in order to reduce time consumption.

Step 3. Calculate $Q(r)$ for each block image, $0 \leq r \leq \sqrt{2}(N-$ 1).

Step 4. Find $r^{\prime}$ such that $Q\left(r^{\prime}\right)>Q(r)$ for any $0 \leq r_{\min } \leq$ $r \leq r_{\max } \leq \sqrt{2}(N-1), r \neq r^{\prime}$ (find the position of the largest peak), $r_{\min }, r_{\max }$ denote the possible minimum and maximum value of $r$, respectively. For a 500 dpi fingerprint image, the range of ridge distances is $\{3,25\}$ [6]. Thus, $r_{\min }=$ $\operatorname{round}(32 / 25)=1$, and $r_{\max }=\operatorname{round}(32 / 3)=11$. The sense of $r^{\prime}$ is shown in Figure 3.

Step 5. If there is not such a local maximum of $Q(r)$, the estimation is impossible.

Step 6. Find $r^{\prime \prime}$ such that $Q\left(r^{\prime \prime}\right)>Q(r)$ for any $0 \leq r_{\min } \leq$ $r \leq r_{\max } \leq \sqrt{2}(N-1), r \neq r^{\prime}$ (find the position of the second largest peak).

Step 7. Estimate the ridge distance in the block image, $N / r^{\prime}$, with confidence level

$$
\begin{gathered}
\mathrm{DL}=\frac{\alpha}{Q\left(r^{\prime}\right)} \min \left\{Q\left(r^{\prime}\right)-Q\left(r^{\prime \prime}\right), Q\left(r^{\prime}\right)-\left(r^{\prime}-1\right),\right. \\
\left.Q\left(r^{\prime}\right)-Q\left(r^{\prime}+1\right)\right\},
\end{gathered}
$$

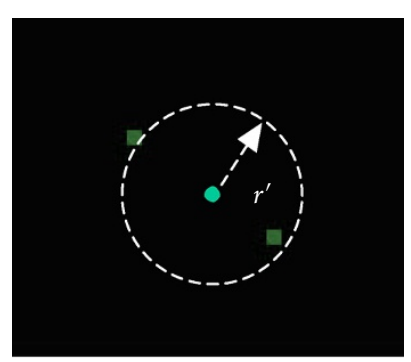

FIGURE 3: The sense of $r^{\prime}$ of a block image in the frequency field.

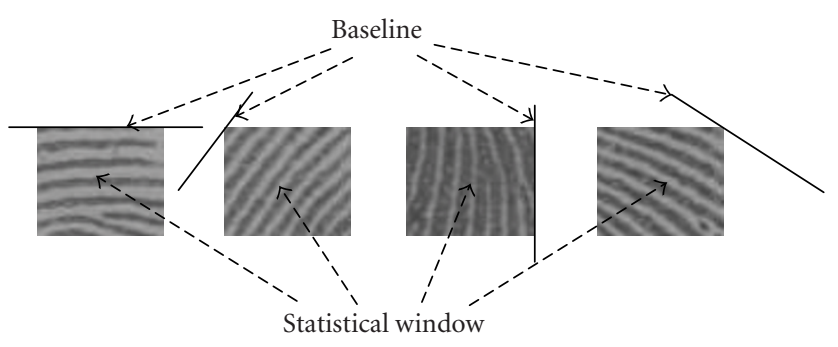

FIGURE 4: Definitions of statistical window and baseline.

where $\alpha=2.43$ (experimental data). Only estimations whose $\mathrm{DL} \geq 0.37$ (experimental data) are accepted.

\section{STATISTICAL METHOD}

A novel and efficient method, called the statistical method, is introduced to estimate ridge distance in fingerprint images in this paper. This method took the following steps.

Step 1. Calculate ridge directions at block level. Divide a fingerprint image into nonoverlap blocks of size $N \times N$ $(N=32)$ and calculate ridge direction for each block by an LMS method [6], which acquires the dominant direction of a block image and views it as the direction of the block. Smooth directions at block level; the initial values of ridge distances of all blocks are assigned to 0 .

Step 2. Use a locally adaptive method [9] to binarize a fingerprint image at block level so that a value of 1 is assigned to pixels on ridges and 0 is assigned to pixels on furrows.

Step 3. Define the statistical window and baseline. Consider each block image of size $N \times N(N=32)$ as a statistical window. The baseline is defined as a beeline parallel to the ridge direction of the block image. The baseline passes the left-top point of the statistical window when the ridge direction of the block image belongs to $[0, \pi / 2)$ and passes the right-top point of the statistical window when the ridge direction of the block image belongs to $[\pi / 2, \pi)$. The definitions of statistical window and baseline are illustrated in Figure 4.

Step 4. Determine the ridge distance distribution in each block image with the distance statistical method. For each statistical window, define an integral array $\mathrm{SA}[\mathrm{MD}+1]$ as a 


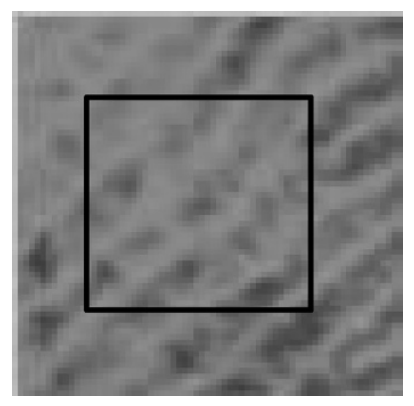

(a)

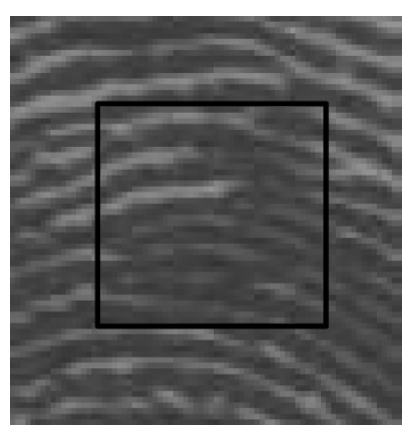

(d)

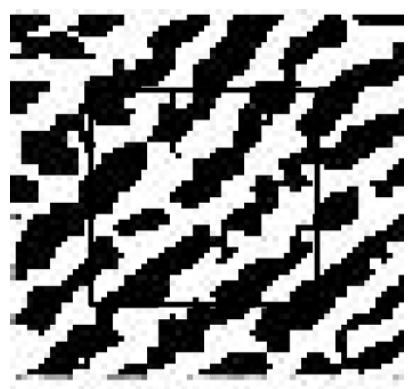

(b)

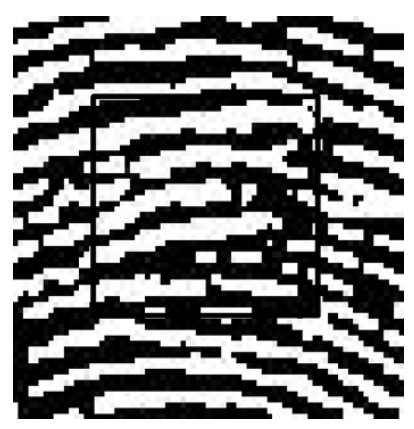

(e)

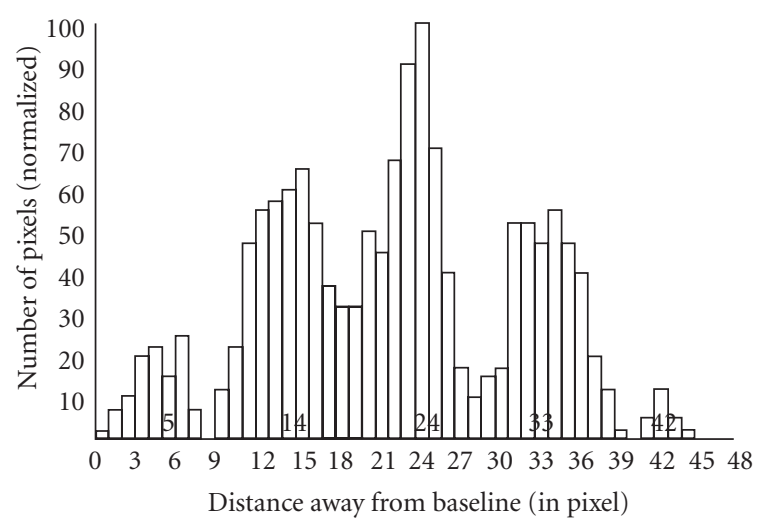

(c)

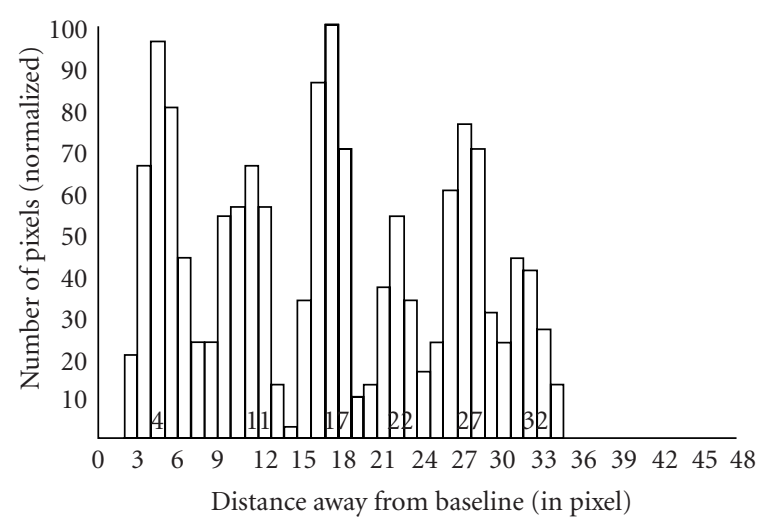

(f)

FIGURE 5: Binarized results: the distribution of ridge distances and peak positions detected in two typical block images: (a) and (d) two typical block images, the inner squares indicate statistical windows; (b) and (e) binarized results for (a) and (d), respectively; (c) and (f) the distribution of ridge distances of (b) and (e) and peaks detected, respectively.

statistical array in which MD denotes the maximum possible distance between pixels on ridges in a statistical window to the baseline. For example, SA $[4]=20$ means there are 20 pixels on ridges in the statistical window 4 pixels away from the baselines. Using $d$ as the $X$-coordinate and SA[d] as the $Y$-coordinate, a histogram of ridge distance distribution in a block image is acquired.

Step 5. Detect positions of all peaks and calculate the peak intervals in the histogram. To each integer $i$ which belongs to $[2, \mathrm{MD}-3]$, if

$$
\begin{aligned}
& \mathrm{SA}[i-1]+\mathrm{SA}[i]+\mathrm{SA}[i+1] \\
&>\mathrm{SA}[i-2]+\mathrm{SA}[i-1]+\mathrm{SA}[i], \\
& \mathrm{SA}[i-1]+\mathrm{SA}[i]+\mathrm{SA}[i+1] \\
&>\mathrm{SA}[i]+\mathrm{SA}[i+1]+\mathrm{SA}[i+2]
\end{aligned}
$$

are satisfied, then $i$ is the position of a peak. Obviously, every peak position corresponds to a ridge and intervals of consecutive peaks denote ridge distances in a statistical window.
Binarized results, the distribution of ridge distances and peak positions detected, are shown for two typical block images in Figure 5.

Step 6. Estimate the ridge distance and calculate the confidence level. If a peak interval is beyond the range of $3 \sim$ 25 , then it is considered an invalid estimation result and should be discarded. After all invalid estimation results are discarded, if the number of the remainder peak intervals is less than 3, assert that the ridge distance of the block could not be estimated. Otherwise, $M_{0}$ and Var, the average and variance of valid peak intervals, are calculated:

$$
\begin{gathered}
M_{0}=\frac{1}{\text { VPINum }} \sum_{i=0}^{\text {VPINum-1 }} \operatorname{VPI}[i], \\
\text { Var }=\frac{1}{\text { VPINum }-1} \sqrt{\sum_{i=0}^{\text {VPINum-1 }}\left(\operatorname{VPI}[i]-M_{0}\right)^{2},}
\end{gathered}
$$

where VPINum is the number of valid peak intervals, and 
$\mathrm{VPI}[i]$ is the $i$ th valid peak interval. The confidence level of estimation result of a block image is defined as follows:

$$
\alpha=1-\frac{\operatorname{Var}}{M_{0}} .
$$

If $\alpha$ is greater than or equal to 0.80 (experimental threshold), the estimation result is accepted and recorded.

\section{HYBRID METHOD}

During the course of implementing the spectral analysis method and the statistical method, the following performance characteristics were observed.

\section{Spectral analysis method}

(1) Not sensitive to ridge directions. Even in regions whose ridge directions vary acutely, for example, regions containing singularities, this method still can estimate ridge distance reliably so long as the quality of the fingerprint image is good.

(2) Sensitive to image quality. When the quality of the fingerprint image is poor, its performance will deteriorate rapidly and the ridge distances of many block images could not be estimated.

(3) Complicated and time consuming.

(4) Estimation results are correspondingly coarse.

\section{Statistical method}

(1) Sensitive to ridge directions. The method performs poorly when ridge directions in the statistical window are very different, especially in regions containing singularities.

(2) Not sensitive to image quality, such as gray-level contrast, or appearance of ridge breaks. Ridge distances can be estimated reliably even if quality of the fingerprint image is poor, if only the dominant direction of a block image is existent and there is no serious ridge conglutination.

(3) Simple and efficient.

(4) Estimation results are correspondingly accurate.

\section{Presentation of hybrid method}

Based on the above analysis of performance of the two methods, a hybrid method that uses the two methods synthetically is defined. First, ridge distances are estimated with the statistical method. For block images that cannot be estimated, the spectral analysis method is then applied. For block images whose ridge distances still cannot be estimated, whose confidence level of estimation result is less than 0.8 , ridge distance of the block is regarded as nonestimable.

\section{PERFORMANCE EVALUATION AND EXPERIMENTAL RESULTS}

It is important to use proper parameters to evaluate performance of methods of ridge distance estimation, which is called performance evaluation. Though the problem has been discussed in [6], further development is still necessary.

\section{Definition of evaluation parameters}

In this paper, we attempt to evaluate the performance of the above three methods by first defining the following parameters.

(1) Direct estimation ratio (DER):

$$
\mathrm{DER}=\frac{A_{d}}{A_{t}} \times 100 \%,
$$

where $A_{d}$ is the area of a fingerprint image for which ridge distance can be estimated in numbers of block images, and $A_{t}$ is the total area of valid fingerprint region in numbers of block images.

DER clearly measures robustness of a method for ridge distance estimation in fingerprint images. A high DER value means that the method is flexible and insensitive to a variety of image quality and ridge directions.

(2) Estimation accuracy (EA): there is always deviation between the estimation result and the actual value of the ridge distance. A small deviation indicates that the estimation result is accurate. Suppose the number of valid blocks in a fingerprint image is $S$ and let $E_{n}$ and $T_{n}$ denote the estimation result and the manually measured value of ridge distance of the $n$th valid block image, respectively; EA is defined as follows:

$$
\mathrm{EA}=\left(1-\frac{1}{S} \sum_{n=1}^{S} \frac{T_{n}-E_{n}}{T_{n}}\right) \times 100 \%
$$

Only block images whose ridge distances can be estimated are considered in the calculation of EA. Here the manually measured value of the ridge distance is used as the actual value. The manually measured value is acquired by measuring the enlarged fingerprint image printed on a piece of paper.

(3) Time consumption (TC): TC is the average time needed for handling one fingerprint image.

\section{Evaluation strategy and experimental conditions}

Methods of ridge distance estimation may show different performance according to the variation in quality of fingerprint images. It is therefore necessary to implement performance evaluation on good, fair, and poor quality images. An average performance of methods on all test images should also be presented.

Until now, it has been difficult to find quantitative methods for strict measurement of the quality of fingerprint images. Considering mainly factors such as gray-level contrast, ridge break, ridge conglutination, and so forth, we selected 30 typical images (10 good-quality, 10 fair-quality, and 10 poor-quality) from the NJU fingerprint database (1200 live scan images, 10 per individual, with image resolutions $=450 \mathrm{dpi}$ and image size $=320 \times 320)$ according to experience to the estimate ridge distance with each of the 


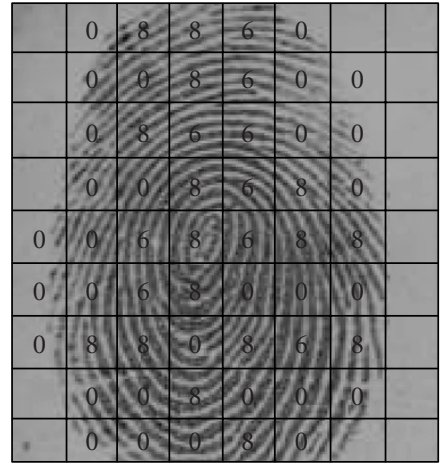

(a)

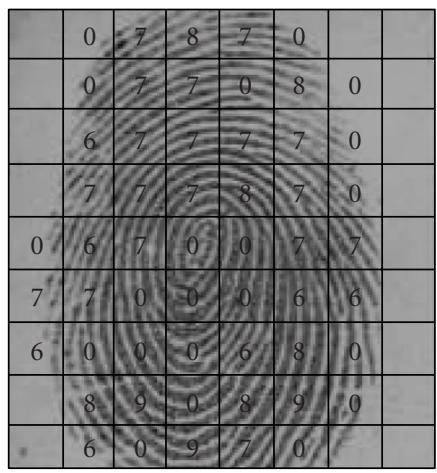

(d)

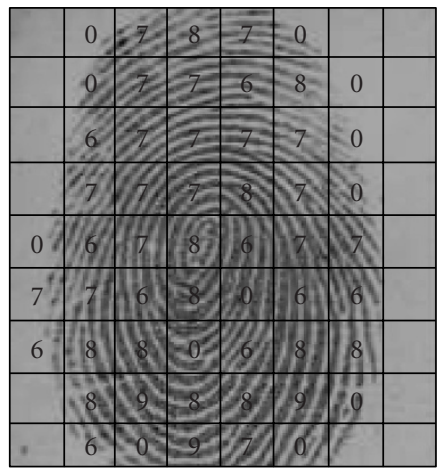

(g)

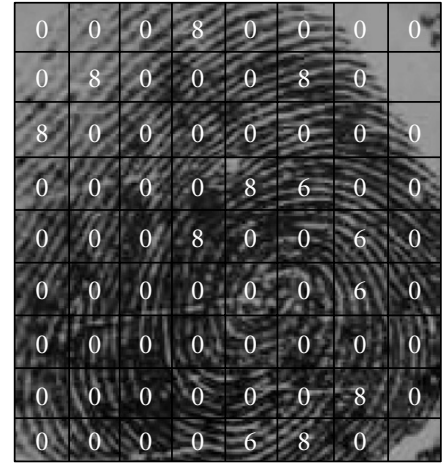

(b)

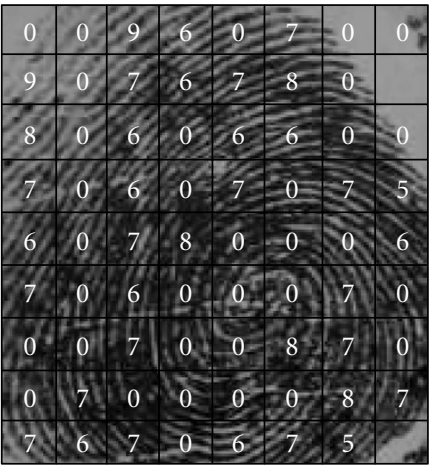

(e)

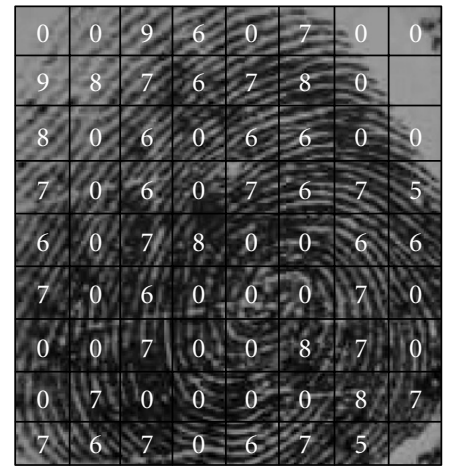

(h)

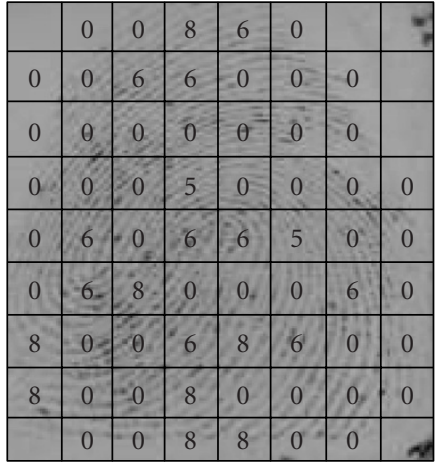

(c)

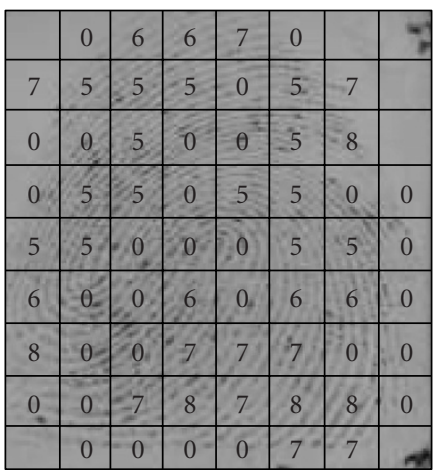

(f)

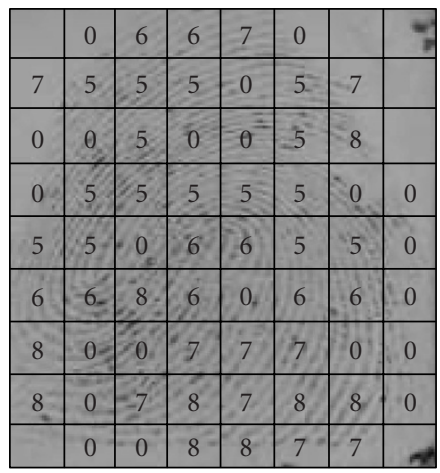

(i)

FIGURE 6: Results of ridge distance estimation with three methods: estimation values are overlapped on images based on (a), (b), and (c) spectral analysis method; (d), (e), and (f) statistical method; (g), (h), and (i) hybrid method.

traditional spectral analysis method, the statistical method, and the hybrid method. Sizes of block images are all $32 \times 32$ and values of the ridge distance in each block image were manually measured. The evaluation is performed with a computer Pentium 2.0 G, 256 M RAM.

\section{Experimental results}

DER, EA, and TC of the three methods on 10 good-quality images, 10 fair-quality images, 10 poor-quality images, and total 30 fingerprint images are listed in Tables 1, 2, 3, and 4, respectively. Estimation results on some typical fingerprint images with the three methods are shown in Figure 6.

\section{CONCLUSION AND DISCUSSION}

We have applied traditional spectral analysis method for the estimation of ridge distances in fingerprint images. A new method, the statistical method, is also evaluated in this paper. For good-quality fingerprint images, there is no dramatic difference in performance between the spectral analysis method 
TABLE 1: Performance of the three methods on 10 good-quality images.

\begin{tabular}{lccc}
\hline \multicolumn{1}{c}{ Methods } & DER & EA & TC \\
\hline Spectral analysis method & $75.2 \%$ & $92 \%$ & $0.42 \mathrm{~s}$ \\
Statistical method & $74.6 \%$ & $97 \%$ & $0.31 \mathrm{~s}$ \\
Hybrid method & $95.5 \%$ & $95 \%$ & $0.32 \mathrm{~s}$ \\
\hline
\end{tabular}

TABle 2: Performance of the three methods on 10 fair-quality images.

\begin{tabular}{lccc}
\hline \multicolumn{1}{c}{ Methods } & DER & EA & TC \\
\hline Spectral analysis method & $38.2 \%$ & $83 \%$ & $0.42 \mathrm{~s}$ \\
Statistical method & $69.3 \%$ & $94 \%$ & $0.31 \mathrm{~s}$ \\
Hybrid method & $88.1 \%$ & $91 \%$ & $0.33 \mathrm{~s}$ \\
\hline
\end{tabular}

and the statistical method (Table 1). For fair and poorquality fingerprint images, however, performance of the statistical method is superior to that of the spectral analysis method (Tables 2 and 3). The statistical method is in addition more efficient. The statistical method shows better average performance on the total 30 images (Table 4). The obvious disadvantage of the statistical method is that it performs poorly in regions where there is acute variation of ridge directions.

Based on analysis and comparison of the performance of the two methods, a hybrid method is developed in this paper. In this method, the statistical method is used as the primary method for regions where ridge direction varies gently; the spectral analysis method is used as a supplement. The advantages of the statistical method and the spectral analysis method are thereby combined. Experimental results show that the hybrid method performs better than either single method, especially in achieving high overall DER (Table 4).

The key weakness of the spectral analysis method is how to determine $r^{\prime}$ accurately and reliably. If this problem can be solved, the performance of the spectral analysis method will be significantly improved. Statistical method depends heavily on reliable determination of ridge direction, especially when the fingerprint image quality is poor.

Window methods, which estimate ridge distance within block images, have intrinsic limitations. For example, the size of block images is a compromise between two factors: big windows may contain ridges with high curvature, causing difficulty in the estimation of distances; if small windows are used, there are too few ridges within a window to estimate the ridge distances. Our future research will focus on a regionbased method that divides a fingerprint image into several regions in which ridge directions are approximately the same and ridge distances are estimated at the regional level.

\section{REFERENCES}

[1] Y. L. Yin, X. B. Ning, and X. M. Zhang, "Development and application of automatic fingerprint identification technology," J. Nanjing Univ. Natur. Sci., vol. 38, no. 1, pp. 29-35, 2002.
TABle 3: Performance of the three methods on 10 poor-quality images.

\begin{tabular}{lccc}
\hline \multicolumn{1}{c}{ Methods } & DER & EA & TC \\
\hline Spectral analysis method & $20.7 \%$ & $77 \%$ & $0.42 \mathrm{~s}$ \\
Statistical method & $47.5 \%$ & $88 \%$ & $0.31 \mathrm{~s}$ \\
Hybrid method & $58.2 \%$ & $87 \%$ & $0.37 \mathrm{~s}$ \\
\hline
\end{tabular}

TABle 4: Performance of the three methods on total of 30 fingerprint images.

\begin{tabular}{lccc}
\hline \multicolumn{1}{c}{ Methods } & DER & EA & TC \\
\hline Spectral analysis method & $44.7 \%$ & $84 \%$ & $0.42 \mathrm{~s}$ \\
Statistical method & $63.8 \%$ & $93 \%$ & $0.31 \mathrm{~s}$ \\
Hybrid method & $80.6 \%$ & $91 \%$ & $0.34 \mathrm{~s}$ \\
\hline
\end{tabular}

[2] L. Hong, A. Jain, S. Pankanti, and R. Bolle, "Identity authentication using fingerprints," in Proc. 1st International Conference on Audio- and Video-Based Biometric Person Authentication, pp. 103-110, Switzerland, March 1997.

[3] L. O'Gorman and J. V. Nickerson, "An approach to fingerprint filter design," Pattern Recognition, vol. 22, no. 1, pp. 29-38, 1989.

[4] W.-C. Lin and R. C. Dubes, "A review of ridge counting in dermatoglyphics," Pattern Recognition, vol. 16, no. 1, pp. 1-8, 1983.

[5] D. C. Douglas Hung, "Enhancement and feature purification of fingerprint images," Pattern Recognition, vol. 26, no. 11, pp. 1661-1671, 1993.

[6] L. Hong, Y. Wan, and A. K. Jain, "Fingerprint image enhancement: algorithm and performance evaluation," IEEE Trans. on Pattern Analysis and Machine Intelligence, vol. 20, no. 8, pp. 777-789, 1998.

[7] Zs. M. Kovacs-Vajna, R. Rovatti, and M. Frazzoni, "Fingerprint ridge distance computation methodologies," Pattern Recognition, vol. 33, no. 1, pp. 69-80, 2000.

[8] D. Maio and D. Maltoni, "Ridge-line density estimation in digital images," in Proc. 14th International Conference on Pattern Recognition, vol. 1, pp. 534-538, Brisbane, Australia, August 1998.

[9] J. Bernsen, "Dynamic thresholding of grey-level images," in Proc. 8th International Conference on Pattern Recognition, pp. 1251-1255, Paris, France, 1986.

Yilong Yin was born in China in 1972. He received his Ph.D. degree from Jilin University, Changchun, China, in 2000. From September 2000 to August 2002, he worked as a Postdoctor in electronic science and engineering, Nanjing University, China. Since September 2002, he has been an Associate Professor in the Department of Computer Science and Technology, Shandong University, China. His research interests are in bio-

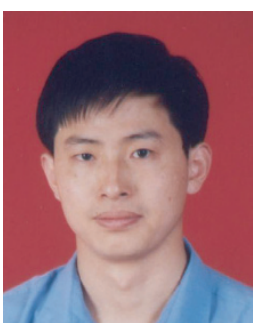
metrics, image processing, pattern recognition, machine learning, and so forth, and now he mainly focuses on robust fingerprint identification algorithm. He has published more than 20 research papers in academic journals and international conferences and he is a member of Special Committee on Machine Learning of China. 
Jie Tian received his Ph.D. degree (with honor) in artificial intelligence from the Institute of Automation, Chinese Academy of Sciences, in 1992. During the years from 1994 to 1996, he was a postdoctoral fellow at the medical image processing group, University of Pennsylvania. Since 1997, he has been a Professor in the Institute of Automation, Chinese Academy of Sciences. His research interests are in the bioinformatics,

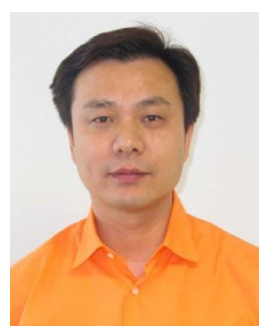
medical image process and analysis, pattern recognition, and so forth. He has published more than 50 research papers in academic journals and international conferences. Dr. Tian is the Reviewer of Mathematical Reviews, American Mathematical Society. Furthermore, he is the Director of a special social committee of pattern recognition and machine intelligence of Chinese Society of Automation and the Founding Member of a special interest group on nonlinear dynamics (SIG/NL) under International Nonlinear Networks System (INNS).

Xiukun Yang is currently a Research Scientist at Identix Incorporated (Nasdaq: IDNX), the world's leading multi-biometric security technology company located in New Jersey, USA, where she focuses on the development and optimization of fingerprint identification algorithms. After she got her Ph.D. degree from Jilin University, Changchun, China in 1997, Dr. Xiukun Yang came to the US as a Research Asso-

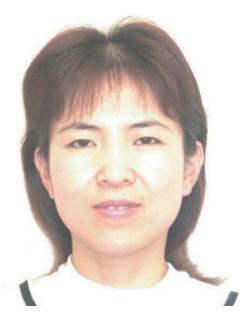
ciate in Michigan State University, where she conducted research on computer vision and artificial intelligence application in automated defect identification of various products, with special emphasis on the development of generic algorithms and neural networks oriented classification and pattern recognition techniques. In April 2000, she started working for August Technology Corporation, headquartered in Bloomington, Minnesota, a leading manufacturer of automated visual inspection solutions for the vast microelectronics industry, where she was mainly involved in the design and implementation of advanced image processing algorithms used in semiconductor wafer macrodefect inspection software system. 